\title{
MAXIMAL TORI IN THE SYMPLECTOMORPHISM GROUPS OF HIRZEBRUCH SURFACES
}

\author{
YAEL KARSHON
}

ABstract. We count the conjugacy classes of maximal tori in the groups of symplectomorphisms of $S^{2} \times S^{2}$ and of the blow-up of $\mathbb{C P}^{2}$ at a point.

Consider the group $\operatorname{Ham}(M, \omega)$ of Hamiltonian symplectomorphisms of a symplectic manifold. ${ }^{1}$ A $k$-dimensional torus in $\operatorname{Ham}(M, \omega)$ is a subgroup which is isomorphic to $\left(S^{1}\right)^{k}$. A maximal torus is one which is not contained in any strictly larger torus.

An action of $\left(S^{1}\right)^{k}$ on $(M, \omega)$ is called Hamiltonian if it admits a moment map, i.e., a map $\Phi: M \rightarrow \mathbb{R}^{k}$ such that $d \Phi_{i}=-\iota\left(\xi_{i}\right) \omega$ for all $i=1, \ldots, k$, where $\xi_{1}, \ldots, \xi_{k}$ are the vector fields on $M$ that generate the action.

A Hamiltonian $\left(S^{1}\right)^{k}$-action defines a homomorphism from $\left(S^{1}\right)^{k}$ to $\operatorname{Ham}(M, \omega)$. The action is effective if and only if this homomorphism is one to one. Its image is then a $k$-dimensional torus in $\operatorname{Ham}(M, \omega)$. Every $k$-dimensional torus in $\operatorname{Ham}(M, \omega)$ is obtained in this way, and two Hamiltonian actions give the same torus if and only if they differ by a reparametrization of $\left(S^{1}\right)^{k}$. Tori in $\operatorname{Ham}(M, \omega)$ have dimension at most $\frac{1}{2} \operatorname{dim} M$. A Hamiltonian action of a $\left(\frac{1}{2} \operatorname{dim} M\right)$-dimensional torus is called toric.

Theorem 1. Let $(M, \omega)$ be a compact symplectic four-manifold. Suppose that $\operatorname{dim} H^{2}(M, \mathbb{R}) \leq 3$ and $\operatorname{dim} H^{1}(M, \mathbb{R})=0$. Then every Hamiltonian circle action on $(M, \omega)$ extends to a toric action.

Remark. Many symplectic four-manifolds do not admit Hamiltonian circle actions. The theorem does not say anything about such manifolds.

Two tori, $T_{1}$ and $T_{2}$, in $\operatorname{Ham}(M, \omega)$ are conjugate if there exists an element $g \in \operatorname{Ham}(M, \omega)$ such that $g T_{1} g^{-1}=T_{2}$. Two torus actions, viewed as homomorphisms $\left(S^{1}\right)^{k} \rightarrow \operatorname{Ham}(M, \omega)$, give conjugate tori in $\operatorname{Ham}(M, \omega)$ if and only if they differ by an equivariant symplectomorphism composed with a reparametrization of $\left(S^{1}\right)^{k}$.

Received July 17, 2002.

This research was partially supported by the Israel Science Foundation founded by The Academy of Sciences and Humanities.

2000 Mathematics Subject Classification. Primary 53D35; Secondary 53D20.

${ }^{1}$ Recall, a Hamiltonian symplectomorphism is one which can be connected to the identity by a path $\psi_{t}$ such that $\frac{d}{d t} \psi_{t}=X_{t} \circ \psi_{t}$ and $\iota\left(X_{t}\right) \omega=d H_{t}$ for a smooth $H_{t}: M \rightarrow \mathbb{R}$. 

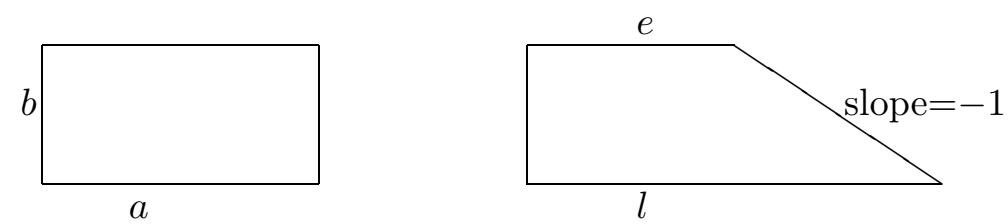

FiguRE 1. Moment map images for standard torus actions on $\left(S^{2} \times S^{2}, \omega_{a, b}\right)$ and $\left(\widetilde{\mathbb{C P}^{2}}, \widetilde{\omega}_{l, e}\right)$.

On $S^{2} \times S^{2}$, let $\omega_{a, b}=a \omega_{1}+b \omega_{2}$, where $\omega_{1}$ and $\omega_{2}$ are the pullbacks through the two projection maps of the rotation invariant area form on $S^{2}$ with total area $2 \pi$. The standard $\left(S^{1}\right)^{2}$-action has the moment map image shown in Figure 1 on the left.

Let $\widetilde{\mathbb{C P}^{2}}$ be the blow-up of $\mathbb{C P}^{2}$ at a point. In it, let $E$ be the exceptional divisor and let $L$ be a $\mathbb{C P}^{1}$ which is disjoint from $E$. For $l>e>0$, let $\widetilde{\omega}_{l, e}$ be a symplectic form such that the symplectic areas of $L$ and $E$ are $2 \pi l$ and $2 \pi e$, respectively. (One can construct $\left(\widetilde{\mathbb{C P}^{2}}, \widetilde{\omega}_{l, e}\right)$ explicitly, e.g., as in [Ka, $\left.\S 6.3\right]$. By [McD2], it is unique up to symplectomorphism.) The standard $\left(S^{1}\right)^{2}$-action (induced from the action on $\mathbb{C P}^{2}$ ) has the moment map image shown in Figure 1 on the right.

Theorem 2. For $a \geq b>0$, the number of conjugacy classes of maximal tori in $\operatorname{Ham}\left(S^{2} \times S^{2}, \omega_{a, b}\right)$ is $\lceil a / b\rceil$.

For $l>e>0$, the number of conjugacy classes of maximal tori in $\operatorname{Ham}\left(\widetilde{\mathbb{C P}^{2}}, \widetilde{\omega}_{l, e}\right)$ is $\left\lceil\frac{e}{l-e}\right\rceil$.

Remark. Here, $\lceil r\rceil$ denotes the smallest integer greater than or equal to $r$. We will prove Theorem 2 by enumerating the different conjugacy classes by the set of integers $k$ that satisfy $0 \leq k<r$ for $r=a / b$ and $r=\frac{e}{l-e}$, respectively.

Remark. The topology of $\operatorname{Ham}\left(S^{2} \times S^{2}, \omega_{a, b}\right)$ also changes when the ratio $a / b$ crosses an integer. See [Gr, Ab, AM, McD3, An]. Also see the remark at the end of the paper.

Remark. There are infinitely many conjugacy classes of tori in the group of contactomorphisms of an overtwisted $S^{3}$ or lens space. See [L1].

For a count of the conjugacy classes of tori in the group of contactomorphisms of the pre-quantum line bundle over a Hirzebruch surface, see [L2].

We recall standard facts about Hamiltonian circle actions on compact symplectic manifolds: By local arguments, each component of the fixed point set is a submanifold of even dimension and has even index. By Morse-Bott theory, each local extremum for the moment map is a global extremum, and these extrema are attained on connected sets. See [GS2, §32]. An interior fixed point is a fixed point which is not a minimum or maximum for the moment map. A fixed surface is a two dimensional connected component of the fixed point set. 
Lemma 1. Let $M$ be a closed symplectic four-manifold with a Hamiltonian circle action. The dimension of $H^{2}(M)$ is equal to the number of interior fixed points plus the number of fixed surfaces. If $\operatorname{dim} H^{1}(M)=0$, each fixed surface has genus zero.

Proof. We apply a standard Morse theory argument. The moment map is a perfect Morse-Bott function whose critical points are the fixed points for the circle action [GS2, §32]. Therefore, $\operatorname{dim} H^{j}(M)=\sum \operatorname{dim} H^{j-i_{F}}(F)$, where we sum over the connected components of the fixed point set, and where $i_{F}$ is the index of the component $F$. The theorem follows by a simple computation of the summands, which is summarized in the table below.

\begin{tabular}{c|c|c|c|c|c|c|c|c|}
\multicolumn{1}{c|}{} & \multicolumn{7}{c}{ Contribution to } \\
\multicolumn{1}{c|}{$\Phi(F)$} & \multicolumn{1}{c}{$i_{F}$} & $H^{0}(M)$ & $H^{1}(M)$ & $H^{2}(M)$ & $H^{3}(M)$ & $H^{4}(M)$ \\
\hline fixed & minimal & 0 & 1 & $2 \operatorname{genus}(F)$ & 1 & & \\
\cline { 2 - 9 } surface & maximal & 2 & & & 1 & $2 \operatorname{genus}(F)$ & 1 \\
\hline $\begin{array}{c}\text { isolated } \\
\text { fixed } \\
\text { point }\end{array}$ & minimal & 0 & 1 & & & & \\
\cline { 2 - 9 } & interior & 2 & & & 1 & & \\
\cline { 2 - 8 } & maximal & 4 & & & & & 1 \\
\hline
\end{tabular}

Proof of Theorem 1. By [Ka, Prop. 5.21], a Hamiltonian circle action extends to a toric action if and only if each fixed surface has genus zero and each nonextremal level set for the moment map contains at most two non-free orbits.

By Lemma 1, since $\operatorname{dim} H^{1}(M)=0$, each fixed surface has genus zero.

By [Ka, Theorem 5.1], if all the fixed points are isolated, the circle action extends to a toric action. Therefore, let us assume that there exists at least one fixed surface. Suppose that the moment map attains its maximum on this surface; the case of a minimum can be treated similarly. By Lemma 1, since $\operatorname{dim} H^{2}(M, \mathbb{R}) \leq 3$, there exist at most two interior fixed points.

A $Z_{k}$-sphere is a 2 -sphere inside $M$ on which the circle acts by rotations with speed $k$. A non-free orbit is either a fixed point or belongs to a $Z_{k}$-sphere. See $[\mathrm{Au}]$ or [Ka, Lemma 2.2]. A $Z_{k}$-sphere intersects each level set in at most one orbit. The north pole of a $Z_{k}$-sphere is an isolated, hence interior, fixed point (because we assume that the maximal set of the moment map is not isolated). Different $Z_{k}$-spheres have different north poles. These considerations show that the number of non-free orbits in an non-extremal level set for the moment map is at most the number of interior fixed points. The theorem follows.

For each non-negative integer $m$, consider the family of trapezoids shown in Figure 2, parametrized by the height $b$ and average width $a>\frac{m}{2} b$. We call these standard Hirzebruch trapezoids. More generally, consider their images under the group $\operatorname{AGL}(2, \mathbb{Z})$ of transformations of $\mathbb{R}^{2}$ of the form $x \mapsto R x+v$ with $R \in \mathrm{GL}(2, \mathbb{Z})$ and $v \in \mathbb{R}^{2}$. These we call Hirzebruch trapezoids. Hirzebruch trapezoids modulo $\mathrm{AGL}(2, \mathbb{Z})$ are in natural one-to-one bijection with the set 


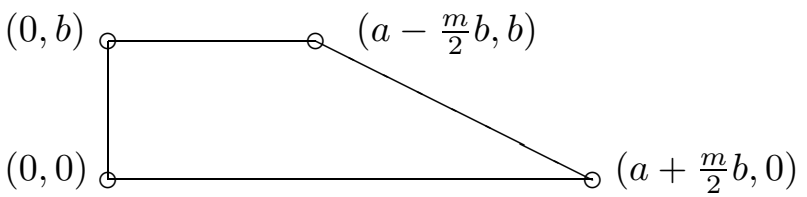

FiguRE 2. A standard Hirzebruch trapezoid
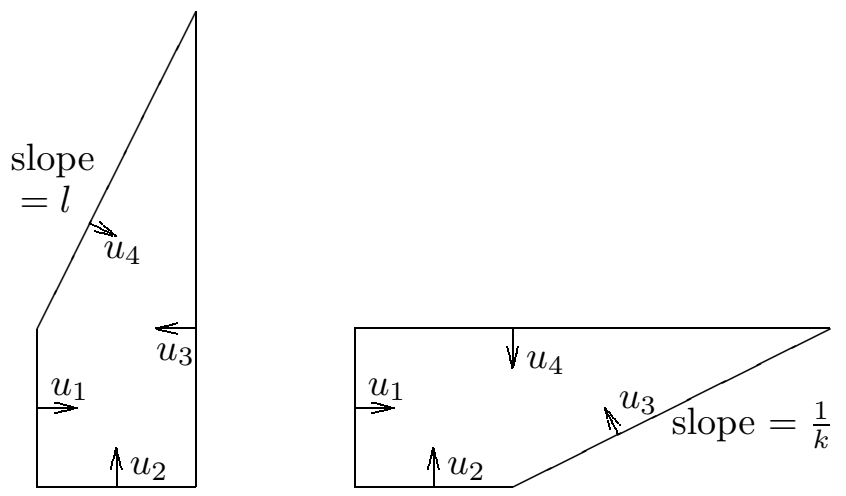

Figure 3. Hirzebruch trapezoids

of parameters $(a, b, m)$ with $m$ a non-negative integer, $a$ and $b$ positive real numbers, and $a>\frac{m}{2} b$.

Take a polygon in $\mathbb{R}^{2}$. Let $u_{1}, \ldots, u_{m}$ be normal vectors to its edges, in counterclockwise order, pointing inward. The polygon is a Delzant polygon if we can choose these normals so that $u_{i} \in \mathbb{Z}^{2}$ and $\operatorname{det}\left(u_{i} u_{i+1}\right)=1$ for all $i$, the indices taken cyclically. ${ }^{2}$

Lemma 2. Every Delzant polygon with four edges is a Hirzebruch trapezoid.

Proof. Fix a Delzant polygon with four edges. Let $u_{1}, u_{2}, u_{3}$, and $u_{4}$ be normals to its edges that satisfy the above Delzant condition. Because $\operatorname{det}\left(u_{1} u_{2}\right)=1$, we may assume, without loss of generality, that $u_{1}=(1,0)$ and $u_{2}=(0,1)$. The conditions $\operatorname{det}\left(u_{2} u_{3}\right)=1$ and $\operatorname{det}\left(u_{4} u_{1}\right)=1$ imply that $u_{3}=(-1, k)$ and $u_{4}=(l,-1)$ for some $k, l \in \mathbb{Z}$. The condition $\operatorname{det}\left(u_{3} u_{4}\right)=1$ then implies $k l=0$. Each of the cases $k=0$ and $l=0$ gives a Hirzebruch trapezoid, as shown in Figure 3.

The convexity theorem of Atiyah, Guillemin and Sternberg, [At,Gu-St], states that, for a Hamiltonian torus action an a compact symplectic manifold, the image of the moment map is a convex polytope. By Delzant's classification of Hamiltonian toric actions $[\mathrm{De}],{ }^{3}$ the moment map images for toric actions are

\footnotetext{
${ }^{2}$ More generally, the Delzant condition for a polytope in $\mathbb{R}^{n}$ is that exactly $n$ facets meet at every vertex and the normals to these facets can be chosen to be generators of $\mathbb{Z}^{n}$.

${ }^{3}$ Also see $[\mathrm{L}-\mathrm{T}]$.
} 

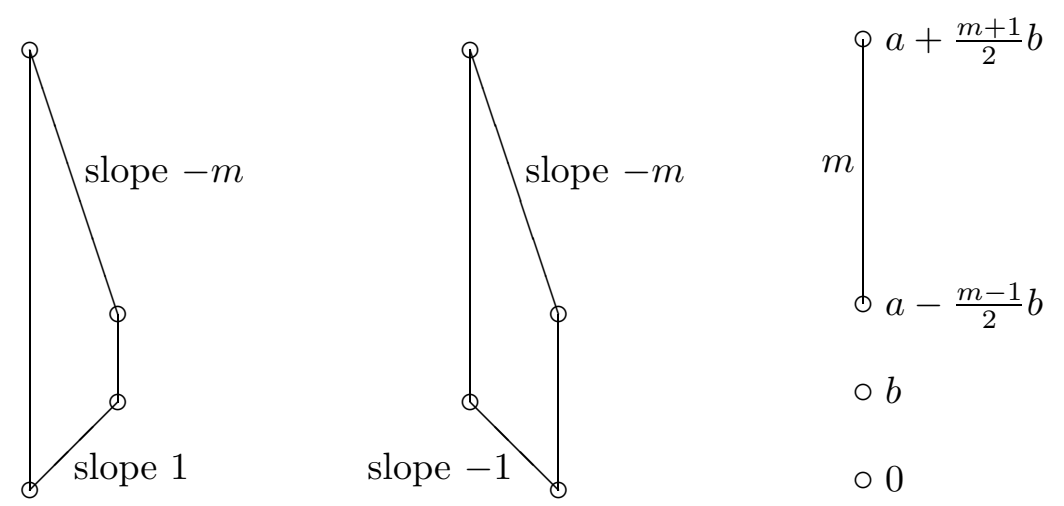

FiguRE 4. Hirzebruch trapezoids with parameters $(m+1, a, b)$ and $(m-1, a, b)$.

precisely the Delzant polytopes, and two toric actions are equivariantly symplectomorphic if and only if their moment map images are translates of each other.

The symplectic four-manifolds that correspond to Hirzebruch trapezoids are Hirzebruch surfaces. See $[\mathrm{Au}]$ or [Ka, section 6.3]. Specifically, when $m=0$ or $m=1$, these are $\left(S^{2} \times S^{2}, \omega_{a, b}\right)$ and $\left(\widetilde{\mathbb{C P}^{2}}, \widetilde{\omega}_{l, e}\right)$, respectively.

Lemma 3. A Hirzebruch surface which corresponds to an integer $m^{\prime} \geq 2$ is symplectomorphic to the Hirzebruch surface which corresponds to the integer $m^{\prime}-2$ with the same parameters $a$ and $b$.

Proof. Take two Hirzebruch trapezoids with the same parameters $a$ and $b$, corresponding to the integers $m^{\prime}$ and $m^{\prime}-2$. After transforming by appropriate elements of $\operatorname{AGL}(2, \mathbb{Z})$, they can be brought to the forms shown in Figure 4 , with $m=m^{\prime}-1$.

In $[\mathrm{Ka}, \S 2.1]$ we associate a labeled graph to every Hamiltonian $S^{1}$-space, such that two spaces are isomorphic if and only if their graphs are isomorphic [Ka, Theorem 4.1]. If the $S^{1}$-action is obtained by restriction of a toric action, the graph can be easily read from the Delzant polygon. See [Ka, §2.2]. The two polygons in Figure 4 give rise to the same graph (shown in Figure 4 on the right). Therefore, the spaces are $S^{1}$-equivariantly symplectomorphic.

Remark. Lemma 3 (which I learned from S. Tolman) seems to be well known. For instance, the new circle action on $S^{2} \times S^{2}$ obtained by identifying this space with the Hirzebruch surface with parameters $(a, b, m=2)$ plays a role in [McD1].

Lemma 4. Among the symplectic manifolds $\left(S^{2} \times S^{2}, \omega_{a, b}\right)$, for $a \geq b>0$, and $\left(\widetilde{\mathbb{C P}^{2}}, \widetilde{\omega}_{l, e}\right)$, for $l>e>0$, no two are symplectomorphic.

Proof. The manifolds $S^{2} \times S^{2}$ and $\widetilde{\mathbb{C P}^{2}}$ are not homeomorphic. For instance, the self intersection of the exceptional divisor in $\widetilde{\mathbb{C P}^{2}}$ is -1 whereas in $S^{2} \times S^{2}$ every class in $\mathrm{H}_{2}$ has an even self intersection. 
A diffeomorphism of $S^{2} \times S^{2}$ acts on $H^{2}\left(S^{2} \times S^{2}\right)=\mathbb{Z}^{2}$ by a $2 \times 2$ matrix of integers, with determinant \pm 1 , which preserves the intersection form $\left[\begin{array}{ll}0 & 1 \\ 1 & 0\end{array}\right]$. There are exactly four matrices with this property; they are

$$
\pm\left[\begin{array}{ll}
1 & 0 \\
0 & 1
\end{array}\right] \text { and } \pm\left[\begin{array}{ll}
0 & 1 \\
1 & 0
\end{array}\right]
$$

These cannot take $\omega_{a, b}$ to a different $\omega_{a^{\prime}, b^{\prime}}$ with $a \geq b>0$ and $a^{\prime} \geq b^{\prime}>0$.

A diffeomorphism of $\widetilde{\mathbb{C P}^{2}}$ acts on $H_{2}\left(\widetilde{\mathbb{C P}^{2}}\right)=\mathbb{Z} L \oplus \mathbb{Z} E \cong \mathbb{Z}^{2}$ by a $2 \times$ 2 matrix of integers, with determinant \pm 1 , which preserves the intersection form $\left[\begin{array}{rr}1 & 0 \\ 0 & -1\end{array}\right]$. There are exactly four matrices with this property; they are $\left[\begin{array}{rr} \pm 1 & 0 \\ 0 & \pm 1\end{array}\right]$. These cannot take $\widetilde{\omega}_{l, e}$ to a different $\widetilde{\omega}_{l^{\prime}, e^{\prime}}$ with $l>e>0$ and $l^{\prime}>e^{\prime}>0$.

Proof of Theorem 2. By Theorem 1, we only need to consider two dimensional tori.

The conjugacy classes of 2-tori in $\operatorname{Ham}(M, \omega)$ for all possible symplectic fourmanifolds $(M, \omega)$ are given by all the Delzant polygons in $\mathbb{R}^{2}$, modulo $\operatorname{AGL}(2, \mathbb{Z})$ congruence. This follows immediately from Delzant's classification of Hamiltonian toric actions [De] and the fact that a reparametrization of the 2-torus $S^{1} \times S^{1}$ transforms the moment map image by an element of $\mathrm{GL}(2, \mathbb{Z})$.

Hence, to find the conjugacy classes of 2-tori in the symplectomorphism group of a particular symplectic four-manifold $(M, \omega)$, we must identify which Delzant polygons correspond to a space which is symplectomorphic to $(M, \omega)$, and we must take these polygons modulo $\operatorname{AGL}(2, \mathbb{Z})$-congruence.

The number of edges of a Delzant polygon is equal to 2 plus the second Betti number of the corresponding space. See Lemma 1 and [Ka, section 2.2]. Therefore, when $M=S^{2} \times S^{2}$ or $M=\widetilde{\mathbb{C P}^{2}}$, we only need to consider Delzant polygons with four edges. By Lemma 2, we only need to consider Hirzebruch trapezoids.

Consider the Hirzebruch trapezoid with parameters $m \geq 0$ and $a \geq b>$ 0. Iterate Lemma 3. If $m$ is even, the corresponding Hirzebruch surface is symplectomorphic to $\left(S^{2} \times S^{2}, \omega_{a, b}\right)$. If $m$ is odd, it is symplectomorphic to $\left(\widetilde{\mathbb{C P}^{2}}, \widetilde{\omega}_{l, e}\right)$, where $l=a+\frac{b}{2}$ and $e=a-\frac{b}{2}$, so that the corresponding trapezoid (shown in Figure 1 on the right) still has height $b$ and average width $a$.

Numbers $a \geq b$ can occur as the average width and the height of a Hirzebruch trapezoid with integer parameter $m \geq 0$ if and only if $\frac{a}{b}>\frac{m}{2}$. When $m=2 k$ is even, this becomes the condition $k<\frac{a}{b}$. When $m=2 k+1$ is odd, it becomes the condition $k<\frac{e}{l-e}$, with $l$ and $e$ as above.

We have found the required number of distinct torus actions on each of the symplectic manifolds $\left(S^{2} \times S^{2}, \omega_{a, b}\right), a \geq b>0$, and $\left(\widetilde{\mathbb{C P}^{2}}, \widetilde{\omega}_{l, e}\right), l>e>0$. By 
Lemma 4, this accounts for all possible toric actions on each of these symplectic manifolds.

Remark. To each non-negative integer $m$ we have associated a torus action on $S^{2} \times S^{2}$ if $m$ is even and on $\widetilde{\mathbb{C P}^{2}}$ if $m$ is odd, for appropriate ranges of values of symplectic forms. Each of these torus actions is in fact obtained from an action of a larger, non-abelian, compact Lie group by restricting to its maximal torus. For instance, the standard actions of $\mathrm{SO}(3) \times \mathrm{SO}(3)$ on $S^{2} \times S^{2}$ and of $U(2)$ on $\widetilde{\mathbb{C P}^{2}}$ restrict to the torus actions that correspond to the integers $m=0$ and $m=1$, respectively. More generally, given a non-negative integer $m$, consider the quotient of $S^{3} \times S^{2}$ by the circle action $\lambda:(z, p) \mapsto\left(z \lambda, \lambda^{m} \cdot p\right)$, where $S^{1}$ is the circle group of complex numbers of norm one, $\lambda \in S^{1}$ acts on $z \in S^{3} \subset \mathbb{C}^{2}$ by scalar multiplication, and $p \mapsto \lambda \cdot p$ is the circle action on $S^{2} \subset \mathbb{R}^{3}$ by rotations. This space admits natural Kähler structures with which it is symplectomorphic (but not biholomorphic) to $S^{2} \times S^{2}$ if $m$ is even and to $\widetilde{\mathbb{C P}^{2}}$ if $m$ is odd, with appropriate symplectic forms. On this space there is a natural action of the quotient of $U(2) \times S^{1}$ by the subgroup $\left\{\left(a I, a^{m}\right) \mid a \in S^{1}\right\}$, where $I \in U(2)$ is the identity matrix. Note that this quotient is a central extension of $\mathrm{SO}(3)$ by $S^{1}$. The torus action that corresponds to the integer $m$ comes from this action.

In this context we recall that the only four dimensional compact symplectic $\mathrm{SO}(3)$ manifolds are $S^{2} \times S^{2}$ and $\mathbb{C P}^{2}$, by [I] .

We also recall that the Hamiltonian symplectomorphism group of $S^{2} \times S^{2}$ with equal areas of the two factors retracts to $\mathrm{SO}(3) \times \mathrm{SO}(3)$ by $[\mathrm{Gr}]$ and that the aforementioned compact Lie subgroups of the Hamiltonian symplectomorphism groups of $S^{2} \times S^{2}$ and of $\widetilde{\mathbb{C P}^{2}}$ in some sense carry an essential part of the topology of these symplectomorphism groups [Ab, AM, McD3].

\section{Acknowledgement}

I thank Sue Tolman for calling my attention, years ago, to the argument of Lemma 3. I thank F. Lalonde for calling my attention, also years ago, to the question of finding different actions on the same symplectic manifold. I thank an anonymous referee for helpful comments. I thank Jean-Claude Hausmann for correcting my normalization conventions. I thank Eugene Lerman for helpful comments and for encouraging me to publish this paper.

\section{References}

[Ab] M. Abreu, Topology of symplectomorphism groups of $S^{2} \times S^{2}$, Invent. Math. 131 (1998), 1-23.

[AM] M. Abreu, D. McDuff, Topology of symplectomorphism groups of rational ruled surfaces, in J. Amer. Math. Soc. 13 (2000), 971-1009.

[An] S. Anjos, Homotopy type of symplectomorphism groups of $S^{2} \times S^{2}$, preprint: math.SG/0009220

[At] M. Atiyah, Convexity and commuting Hamiltonians, Bull. London Math. Soc. 14 (1982), 1-15. 
[Au] M. Audin, The topology of torus actions on symplectic manifolds, Progr. Math., 93. Birkhäuser Verlag, Basel, 1991.

[De] T. Delzant, Hamiltoniens périodiques et image convexe de l'application moment, Bull. Soc. Math. France 116 (1988), 315-339.

[Gr] M. Gromov, Pseudoholomorphic curves in symplectic manifolds, Invent. Math. 82 (1985), 307-347.

[GS1] V. Guillemin, S. Sternberg, Convexity properties of the moment mapping, Invent. Math. 67 (1982), 491-513.

[GS2] , Symplectic techniques in physics, Cambridge University Press, Cambridge, 1984.

[I] P. Iglesias, Les $\mathrm{SO}(3)$-variétés symplectiques et leur classification en dimension 4, Bull. Soc. Math. France 119 (1991), 371-396.

[Ka] Y. Karshon, Periodic Hamiltonian flows on four dimensional manifolds, Mem. Amer. Math. Soc. 141 (1999), no. 672.

[L1] E. Lerman, Contact cuts, Israel J. Math. 124 (2001), 77-92.

[L2] Maximal tori in the contactomorphism groups of circle bundles over Hirzebruch surfaces, Math. Res. Lett. 10 (2003), 133-144.

[LT] E. Lerman, S. Tolman, Hamiltonian torus actions on symplectic orbifolds and toric varieties, Trans. Amer. Math. Soc. 349 (1997), 4201-4230.

[McD1] D. McDuff, Examples of symplectic structures, Invent. Math. 89 (1987), 13-36.

[McD2] - Remarks on the uniqueness of symplectic blowing up, Symplectic geometry, pp. 157-167, London Math. Soc. Lecture note Ser., 192, Cambridge Univ. Press, Cambridge, 1993.

[McD3] _ Almost complex structures on $S^{2} \times S^{2}$, Duke Math. J. 101 (2000), 135-177.

Institute of Mathematics, The Hebrew University of Jerusalem, Giv'at Ram, JERUSALEM 91904, ISRAEL.

E-mail address: karshon@math.huji.ac.il 